\title{
Postural Control on a Quadruped Robot using lateral tilt: a Dynamical System approach
}

\author{
Luiz Castro \\ Cristina P Santos \\ Miguel Oliveira \\ Auke Ijspeert
}

\begin{abstract}
:
Autonomous adaptive locomotion over irregular terrain is one important topic in robotics research. Postural control, meaning movement generation for robot legs in order to attain balance, is a first step in this direction. In this article, we focus on the essential issue of modeling the interaction between the central nervous system and the peripheral information in the locomotion context. This issue is crucial for autonomous and adaptive control, and has received little attention so far. This modeling is based on the concept of dynamical systems whose intrinsic robustness against perturbations allows for an easy integration of sensory-motor feedback and thus for closed-loop control. Herein, we focus on achieving balance without locomotion.

The developed controller is modeled as discrete, sensory driven corrections of the robot joint values in order to achieve balance. The robot lateral tilt information modulates the generated trajectories thus achieving balance. The system is demonstrated on a quadruped robot which adjusts its posture until reducing the lateral tilt to a minimum.
\end{abstract}

\section{Introduction}

Autonomous adaptive locomotion over irregular terrain is one important topic in the robotics research. Generating trajectories in autonomous robotics, including legged robots, is still a complex, unsatisfactory solved problem. Despite an intensive research in the field ([3],[7],[1]), adaptation to unpredicted changes is still an open problem. In order to develop autonomous robot systems able to deal with less knowledge of terrain irregularity, it is required a tight coupling of planning, sensing and execution.

The work presented in this article is part of a larger project which aims at developing a closed loop control architecture based on dynamical systems for the autonomous generation, modulation and planning of complex motor behaviors for legged robots with many DOFs. Our approach is partly inspired from the biological concept of CPGs $([4])$ and by the concepts of force fields ([5]) 
found in biology, as we believe this bio-inspiration enables to develop new flexible and robust technical solutions to the locomotion problem. We apply autonomous differential equations to model how behaviors related to locomotion are programmed in the oscillatory feedback systems of CPGs in the nervous systems. These systems are solved using numerical integration.

This dynamical systems approach model for CPGs presents multiple interesting properties comparatively to other methods based on finite-state machines, sine-based trajectories, ZMP-based ([11]) or heuristic control laws such as the Virtual Model Control ([1]). These include: low computation cost which is well-suited for real time; the stability properties of the limit cycle behavior (i.e. perturbations are quickly forgotten); intrinsic robustness against small perturbations; the smooth online modulation of trajectories through changes in the dynamical systems parameters and phase-locking between the different oscillators for different DOFs. Further, these systems, once coupled, produce coordinated multidimensional rhythms of motor activity, under the control of simple input signals.

In order to tackle the complexity inherent to the design of dynamical systems, we choose a modular approach to build our model on the hypothesis that complex movements can be generated through the sequencing and/or superposition of simpler movement primitives implemented as simple, stable discrete and rhythmic dynamical systems. This modular approach is supported by current neurological and human motor control findings. Further, this approach enables to generate rhythmic and discrete movements, as well as their superposition.

As a main application and a first step, we address postural control without locomotion, considering robot lateral tilt information online acquired by accelerometers. Trajectories are modulated online according to these feedback pathways thus achieving balance, i.e, movements for the robot legs are generated in order to reduce this lateral tilt to a minimum. This task involves closed-loop control and we will thus particularly focus on the integration of sensory-motor information in the architecture. The controller is composed of two embedded dynamical discrete and rhythmic modules. The discrete module specifies the offset of the rhythmic movement. In a next step, we will extend this work to the achievement of adaptive quadruped locomotion in unknown, rough terrain that we model as discrete, sensory driven corrections of a basic rhythmic motor pattern for locomotion.

We present results that show how the developed controller successfully generates the required joint movements in order to reduce the lateral tilt.

Control approaches based on CPGs and nonlinear dynamical systems are widely used in robotics to achieve tasks which involve rhythmic motions including autonomous adaptive dynamic walking over irregular terrain ([6],[2]), juggling ([8]), drumming ([9]), and basis field approaches for limb movements ([5]). Quadruped walking control using CPGs exploring sensory feedback integration into the locomotion control has been extensively explored by Hiroshi Kimura and his colleagues. Herein, we address postural control in the framework of dynamical systems with superposition of discrete and rhythmic movements. We build on previous work, where controllers were developed for combining discrete 
and rhythmic motor primitives in drumming and dancing tasks ([9],[10]). In this article, we focus the issue of modeling the interaction between the central nervous system and the peripheral information. This issue is crucial for autonomous and adaptive control, and has received little attention so far. The intrinsic robustness of the dynamical systems approach against perturbations allows for an easy integration of sensory-motor feedback and thus for closed-loop control. The proposed work tries to serve these purposes and focus on the integration of sensory-motor information in the developed dynamical architecture.

In this article, we will first present the dynamical systems to model the rhythmic and discrete movements. In Section III, we present how we achieve postural control in an AIBO robot using lateral tilt information by applying the developed dynamical systems. In Section IV, we present the results obtained. We conclude by discussing the main results we obtained and the work we are currently working on.

\section{Dynamical System}

In this section we present our model of the MPG (Motor Pattern Generator) used to generate the trajectories for one DOF. Two motor primitives generate the rhythmic and the discrete movements. The rhythmic movement is turned off such that only postural control is taken care, without locomotion generation. It exists because the system is conceived to generate locomotion in a posteriori phase. The rhythmic movement is generated by an Hopf oscillator. The discrete primitive is generated by a stable differential equation such that it integrates sensory information in the controller that generates the trajectories.

\subsection{Architecture of the MPG}

The control mechanism of a generic MPG is built on the hypothesis that complex movements can be generated through the superimposition and sequencing of simpler motor primitives implemented as a discrete and a rhythmic movement. Discrete movement is incorporated to the final trajectory as an offset of the rhythmic movement. Trajectory is modulated by particular choices of the dynamical control parameters (see [9] and [10] for details).

The MPG for a DOF $i$ is divided in two dynamical subsystems, one generating the discrete part of the movement $\left(y_{\mathrm{i}}\right)$ and another generating the rhythmic part $\left(x_{\mathrm{i}}, z_{\mathrm{i}}\right)$. The generated trajectories, $x_{\mathrm{i}}$, are sent online for each DOF. The lower level control is done by PID controllers.

\subsection{MPG Rhythmic Movement}

To generate the rhythmic movements we apply the following dynamical system

$$
\begin{aligned}
\dot{x}_{\mathrm{i}} & =\beta\left(\mu_{\mathrm{i}}-r_{\mathrm{i}}^{2}\right)\left(x_{\mathrm{i}}-y_{\mathrm{i}}\right)-\omega z_{\mathrm{i}} \\
\dot{z}_{\mathrm{i}} & =\beta\left(\mu_{\mathrm{i}}-r_{\mathrm{i}}^{2}\right) z_{\mathrm{i}}+\omega\left(x_{\mathrm{i}}-y_{\mathrm{i}}\right)
\end{aligned}
$$

where $r_{\mathrm{i}}=\sqrt{\left(x_{\mathrm{i}}-y_{\mathrm{i}}\right)^{2}+z_{\mathrm{i}}^{2}}$. 
These eqs. describe an Hopf oscillator, where $\mu_{\mathrm{i}}$ controls the amplitude of the oscillations, $\omega$ and $\beta$ controls the speed of convergence to the limit cycle. This Hopf oscillator contains a bifurcation from a fixed point (when $\mu_{\mathrm{i}}<0$ ) to a structurally stable, harmonic limit cycle with radius $R=\sqrt{\mu_{\mathrm{i}}}$ and relaxation to the limit cycle given by $\frac{1}{2 \beta \mu_{\mathrm{i}}}$, for $\mu_{\mathrm{i}}>0$. The fixed point $x_{\mathrm{i}}$ has an offset given by $y_{\mathrm{i}}$, which is the state variable of the discrete system. Thus, the resulting position $x_{\mathrm{i}}$, modifies according to the $y_{\mathrm{i}}$ variable as specified by the discrete movement. For $\mu_{\mathrm{i}}<0$ the system exhibits a stable fixed point at $x_{\mathrm{i}}=y_{\mathrm{i}}$. This Hopf oscillator describes a rhythmic motion which can be switched on or off by simply setting $\mu_{\mathrm{i}}$ to positive or negative values, respectively. Moreover, the amplitude of the movement is specified by $\mu_{\mathrm{i}}$ and its frequency by $\omega_{\mathrm{i}}$.

Currently, rhythmic motion is switched off by setting $\mu_{\mathrm{i}}$ to a negative value, so that the attractor of the Hopf oscillator is no longer a limit cycle but a fixed point specified by the offset, i.e. by $y$ variable. Relaxation time for the rhythmic system is given by $\frac{1}{2 \beta \mu_{\mathrm{i}}}$ parameter. The $y$ variable evolution will be specified and explained in the next subsection. This easy control of the different patterns generated is an advantage of the proposed architecture.

In Fig. 1, $y$ variable (dashed line) is considered to have constant values during some time intervals. Initially $y=9$, it is decreased to 2 at $t=5.6 \mathrm{~s}$ and at instant $t=11.3 \mathrm{~s} y$ is set to -9 . The resulting $x$ trajectory (solid line) converges asymptotically to the current value of $y$ (dashed line). By modifying on the fly the offset values ( $y$ variable), one can easily modulate the generated trajectories. Whatever the change is, the system converges almost immediately to the new solution of the system. Further, notice the smoothness of the trajectory when the parameters are changed.

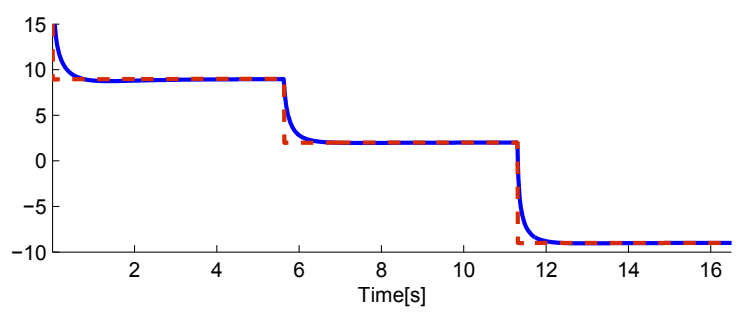

Figure 1: Trajectory modulation through changes in the $y$ values (offset) for dynamical system defined by eqs. 1 and 2 , when rhythmic motion is turned off $\left(\mu_{\mathrm{i}}<0\right)$.

\subsection{MPG Discrete Movement}

To generate the discrete movements, we define a nonlinear dynamical system whose solution, given by $y_{\mathrm{i}}$, is the offset of the output $x_{\mathrm{i}}$ (eq. 1). This discrete system is designed to keep balance, such that by measuring the robot lateral tilt, the controller is able to maintain lateral stability reducing this tilt to a minimum. 
It is important that this discrete movement generator applies to the control of a real robot. Thus, the generated movement must be able to: 1) smoothly adapt to the control parameters and 2) allow trajectory modulation through changes in these control parameters. In our case, the roll is not fixed but changes according to the robot movement during postural control. Therefore, we apply differential equations to model the discrete movement. The discrete movement is generated by the following dynamical system

$$
\dot{y}_{\mathrm{i}}=k_{\mathrm{j}, \mathrm{i}} f(\phi)+\alpha\left(y_{\mathrm{i}}-\mathrm{M}_{\mathrm{i}}\right) e^{-\frac{\left(y_{\mathrm{i}}-\mathrm{M}_{\mathrm{i}}\right)^{2}}{2 \sigma^{2}}}+\alpha\left(y_{\mathrm{i}}-\mathrm{D}_{\mathrm{i}}\right) e^{-\frac{\left(y_{\mathrm{i}}-\mathrm{D}_{\mathrm{i}}\right)^{2}}{2 \sigma^{2}}},
$$

where $\phi$ is the robot lateral tilt, $k_{\mathrm{j}, \mathrm{i}}(\mathrm{j}=$ left, right) is a static gain and $f$ is defined as a linear function of the body's lateral tilt and is given by:

$$
f(\phi)=\left\{\begin{array}{cc}
0, & -0.2<\phi<0.2 \text { (degrees) } \\
0.8 \phi, & \text { elsewhere }
\end{array}\right.
$$

A dead-zone was defined in order to deal with sensor noise. In this dynamical system, two repellors specify joint limits $\left(\mathrm{M}_{\mathrm{i}}\right.$ and $\left.\mathrm{D}_{\mathrm{i}}\right)$. These repellors are multiplied by a Gaussian function which delimits their range of action around the joint limits.

\section{AIBO Postural Control using robot lateral tilt}

In this section we show how we apply the presented MPG to achieve quadruped postural control using lateral tilt of the body on a real ers-7 AIBO robot.

\subsection{Robotic Setup}

We use an AIBO dog robot, which is a 18 DOFs quadruped robot made by Sony. The robot stands up on two platforms, one fixed and another moveable (see Fig. 2(a)). We control the swing and knee of the four AIBO legs, that is 8 DOFs of the robot, 2 DOFs in each leg: elevator and knee joints. For $i=1,3$

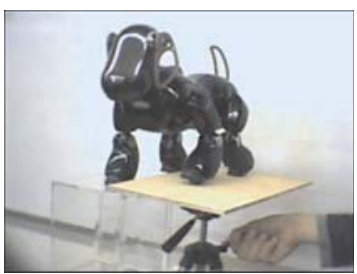

(a)

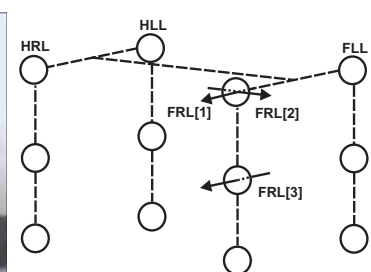

(b)

Figure 2: (a) Real AIBO robot mounted over two platforms, one fixed an the other moveable. (b) Scheme of the AIBO controlled DOFs.

(Swing, Knee) we control FLL[i], FRL[i], for fore legs and HLL[i], HRL[i] for hind legs. The other DOFs are not used for the moment, and remain fixed to an 


\begin{tabular}{l||l|l|l|l|l|l|}
\hline \hline & $\theta_{\text {FLL }}$ FRL & $\theta_{\text {FLL }-H L L}$ & $\theta_{\text {FLL }-H R L}$ & $\theta_{\text {FRL }- \text { HLL }}$ & $\theta_{\text {FRL }-H R L}$ & $\theta_{\text {HLL-HRL }}$ \\
\hline \hline$\left(^{\circ}\right)$ & -180 & -270 & -90 & -90 & 90 & 180 \\
\hline \hline
\end{tabular}

Table 1: Phase differences between swing oscillators (i[1]) used to perform a walking gait.

appropriately chosen value during the experiments. Fig. 2(b) shows a schematic view of the AIBO and the controlled DOFs.

The AIBO has a set of 3-axis accelerometers, built into its body. They enable us to calculate the lateral tilt of the robot body.

\subsection{The Overall Architecture}

We use one generic MPG for each controlled DOF. In order to ensure phaselocked synchronization between the different DOFs of the robot, we couple the different MPGs together. We bilaterally couple the Hopf oscillators of the MPGs, those couplings being illustrated by right-left arrows on fig. 3 and unilaterally couple each swing MPG to the corresponding Knee MPG. For the swing joints, we modify Eqs. 1 and 2 of all the DOFs as follows:

$$
\left[\begin{array}{c}
\dot{x}_{\mathrm{i}[1]} \\
\dot{z}_{\mathrm{i}[1]}
\end{array}\right]=\left[\begin{array}{cc}
\beta \mu_{\mathrm{i}} & \omega \\
-\omega & \beta \mu \mathrm{i}
\end{array}\right]\left[\begin{array}{c}
x_{\mathrm{i}[1]}-y_{\mathrm{i}[1]} \\
z_{\mathrm{i}[1]}
\end{array}\right]-\beta r_{\mathrm{i}[1]}^{2}\left[\begin{array}{c}
x_{\mathrm{i}[1]}-y_{\mathrm{i}[1]} \\
z_{\mathrm{i}[1]}
\end{array}\right]+\sum_{j \neq i} \mathbf{R}\left(\theta_{\mathrm{i}[1]}^{\mathrm{j}[1]}\right)\left[\begin{array}{c}
x_{\mathrm{j}[1]}-y_{\mathrm{j}[1]} \\
z_{\mathrm{j}[1]}
\end{array}\right]
$$

For the knee joints, we modify Eqs. 1 and 2 of all the knee DOFs as follows:

$$
\left[\begin{array}{c}
\dot{x}_{\mathrm{i}[3]} \\
\dot{z}_{\mathrm{i}[3]}
\end{array}\right]=\left[\begin{array}{cc}
\beta \mu \mathrm{i} & \omega \\
-\omega & \beta \mu \mathrm{i}
\end{array}\right]\left[\begin{array}{c}
x_{\mathrm{i}[3]}-y_{\mathrm{i}[3]} \\
z_{\mathrm{i}[3]}
\end{array}\right]-\beta r_{\mathrm{i}[3]}^{2}\left[\begin{array}{c}
x_{\mathrm{i}[3]}-y_{\mathrm{i}[3]} \\
z_{\mathrm{i}[3]}
\end{array}\right]+\frac{1}{2} \mathbf{R}\left(\psi_{\mathrm{i}[3]}^{\mathrm{j}[1]}\right)\left[\begin{array}{c}
x_{\mathrm{j}[1]}-y_{\mathrm{j}[1]} \\
z_{\mathrm{j}[1]}
\end{array}\right]
$$

where $r_{\mathrm{i}}[k]$ is the norm of vector $\left(x_{\mathrm{i}}[k], z_{\mathrm{i}}[k]\right)^{\mathrm{T}}(\mathrm{k}=1,3)$. The linear terms are rotated onto each other by the rotation matrices $\mathbf{R}\left(\theta_{\mathrm{i}[1]}^{\mathrm{j}[1]}\right)$ and $\mathbf{R}\left(\psi_{\mathrm{i}[3]}^{\mathrm{j}[1]}\right)$, where $\theta_{\mathrm{i}[1]}^{\mathrm{j}[1]}$ is the desired relative phase among the $\mathrm{i}[1]$ 's and $\mathrm{j}[1]$ 's MPGs and $\psi_{\mathrm{i}[3]}^{\mathrm{j}[1]}$ is the desired relative phase among the i[3]'s and j[1]'s MPGs (i, j = FLF, FRL, HLL, HRL). In our case, we set these values according to table 1 , which defines the phases required for performing a walking gait (we exploit the fact that $\left.\mathbf{R}(\theta)=\mathbf{R}^{-1}(-\theta)\right)$. The $\psi_{\mathrm{i}[3]}^{\mathrm{j}[1]}$ were all set to $-90^{\circ}$. Due to the properties of this type of coupling among oscillators, the generated trajectories are always smooth and thus potentially useful for real-world implementations such as trajectory generation in a robot.

For offset $y_{\mathrm{i}}$ specification, we measure the lateral tilt of the body, $\phi$. We want to stretch the legs towards which the robot is tilted, and fold the other legs, thus reducing the robot lateral tilt and keeping the body parallel to the ground. This is achieved by reducing the swing and knee joint values of the former and increasing these joint values for the later.

The effect of this offset joint change should be of opposite effect on the left and right legs of the robot, but should also influence similarly both legs. The 
$k_{\mathrm{j}, \mathrm{i}}$ static gain ( $j=$ left, right) (eq. 3$)$ is set symmetrically for the robot left and right legs, and is given by

$$
\begin{aligned}
k_{\text {left }, \mathrm{i}} & =-\mathrm{C}_{\mathrm{i}} \\
k_{\text {right }, \mathrm{i}} & =\mathrm{C}_{\mathrm{i}},
\end{aligned}
$$

where $\mathrm{C}_{\mathrm{i}}$ is set according to the joints range of each leg such that joints change with the same velocity.

The controller architecture is depicted in fig. 3. The MPG generates discrete

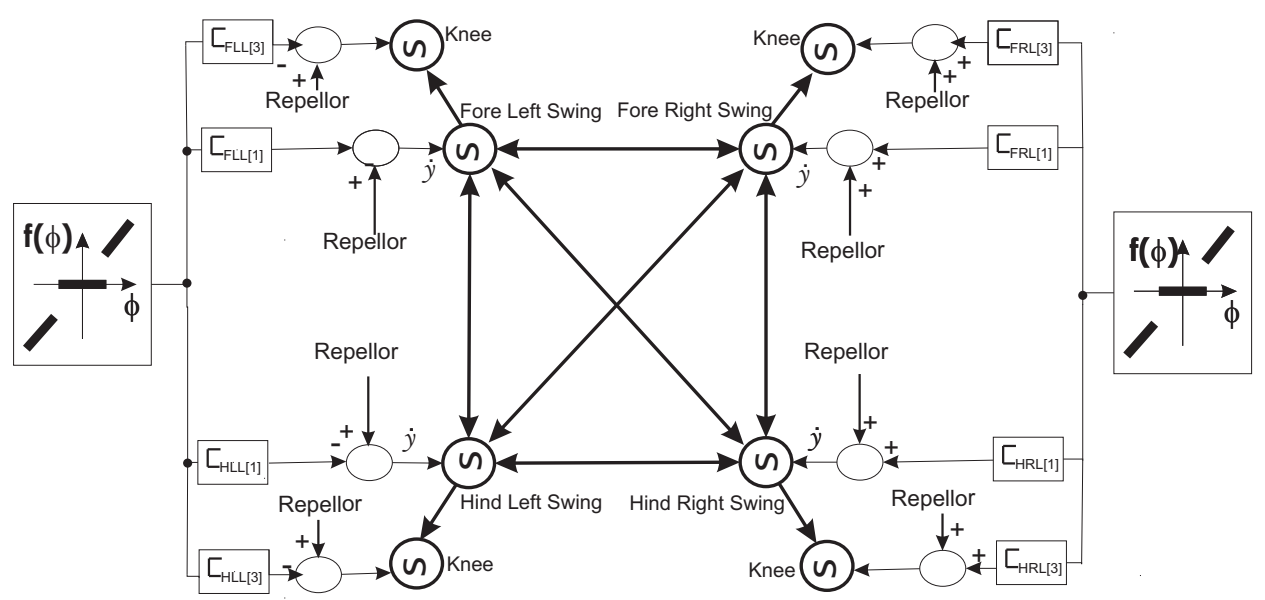

Figure 3: Controller architecture. The lateral tilt value and the joint limits modulate the $y$ and $x$ trajectories.

and rhythmic trajectories, as well as their superposition. Within the MPG, the discrete system specifies an offset for the rhythmic movement. In this particular situation, the rhythmic motion is turned off, so that the attractor of the Hopf oscillator is a fixed point specified by the offset, i.e, by the discrete movement.

Trajectories generated by this architecture are modulated by sensory feedback, according to the lateral tilt of the body. This tilt is linearly transformed by a $f$ function such that it specifies a rate of change for the robot joints. A larger $\phi$ results in stronger rates of change, $\dot{y}_{\mathrm{i}} \cdot y_{\mathrm{i}}$ define the fixed points towards which the MPG Hopf oscillators will converge. The final trajectories $x_{\mathrm{i}}$ specify the planned joint values needed to reduce the lateral tilt to a minimum. These are sent online for each DOF and the lower level control is done by PID controllers. Because motion is sufficiently slow there is no need to apply inverse dynamics.

\section{Results}

In this section, we describe two experiments done in a real AIBO robot. The robot stands in a moveable platform and we forced some changes on the tilt 
of the robot's body. At each sensorial cycle, sensory information is acquired, dynamic equations are calculated and integrated thus specifying servo positions. The robot control loop is measured and has 8 milliseconds. The dynamics of the CPGs are numerically integrated using the Euler method with a fixed time step of $1 \mathrm{~ms}$. Parameters were chosen in order to respect feasibility of the experiment and are given in table 2. We recorded the actual trajectories from the joints incremental encoders $\overrightarrow{\tilde{x}}$ and the planned trajectories $\vec{x}$.

\begin{tabular}{|l|c|c|c|c|c|}
\hline \hline$\beta$ & $\omega\left(\mathrm{rad} s^{-1}\right)$ & $\mu_{\mathrm{i}}$ & $\frac{1}{2 \beta \mu_{\mathrm{i}}}(\mathrm{s})$ & $\left.\alpha\left(s^{-1}\right)\right)$ & $\sigma$ \\
\hline 1.38 & 1.0472 & -36 & 0.1 & 5000 & 0.05 \\
\hline \hline
\end{tabular}

Table 2: Parameter values used in the experiments.

\subsection{First Experiment}

To show the behavior of the system, we start with a very simple experiment, in which the robot is first inclined of $\sim 6^{\circ}$ to its left side and, after sometime, it is again inclined of $\sim 5^{\circ}$ to the same side. We expect the system to react to each of these tilt changes by reducing it to values belonging to a small region around zero, as defined by the dead-zone. Right and left legs are expected to exhibit a symmetric behavior.

In fig. 4 we can see the result of the experiment in the real dog. We depict actual trajectories $\overrightarrow{\tilde{x}}_{\mathrm{FLL}[3]}$ (solid line) and the planned trajectories $\vec{x}_{\mathrm{FLL}[3]}$ (dashed line) of the fore leg knees. The controller reduces considerably the tilt in the first 2 seconds, from $\sim 6^{\circ}$ to $\sim 2^{\circ}$. When the robot is inclined to the left (positive lateral tilt), $\dot{y}_{\mathrm{FLL}[3]}$ is negative (middle panel in the left side of fig. 4), meaning that the offset $y_{\mathrm{FLL}[3]}$ is reduced and the leg extends reducing the tilt. Because at $t=9.4 \mathrm{~s}$ the inclination is slightly smaller than at $t=1 \mathrm{~s}, \dot{y}_{\mathrm{FLL}[3]}$ has a smaller value. Comparing the right and the left knee $\vec{x}_{\mathrm{i}[3]}$ trajectories, we see that the system behave as expected, having symmetric trajectories.

Note that despite the noisy sensorial information, the resultant trajectories are smooth. Further, the sensors are able to follow the planned trajectories as expected.

\subsection{Second Experiment}

In this experiment, the robot is subjected to more abrupt tilt changes and these happen during the controller recover. At $t \sim 4 \mathrm{~s}$ we inclined the robot of $\sim 10^{\circ}$ to the left direction and we expect the system to react to this perturbation by stretching the left legs and folding the right ones, and as such reducing the tilt. The robot is again inclined at $t \sim 5.8 \mathrm{~s}$ to its left side by $\sim 12^{\circ}$, but this change happens before the system had reached a balanced position. Finally, at $t \sim 8 \mathrm{~s}$, the robot is again inclined but towards its right side by $\sim 8^{\circ}$. We expect the system to react to this change in order to reach the equilibrium. Further, right and left legs are expected to exhibit a symmetric behavior. 

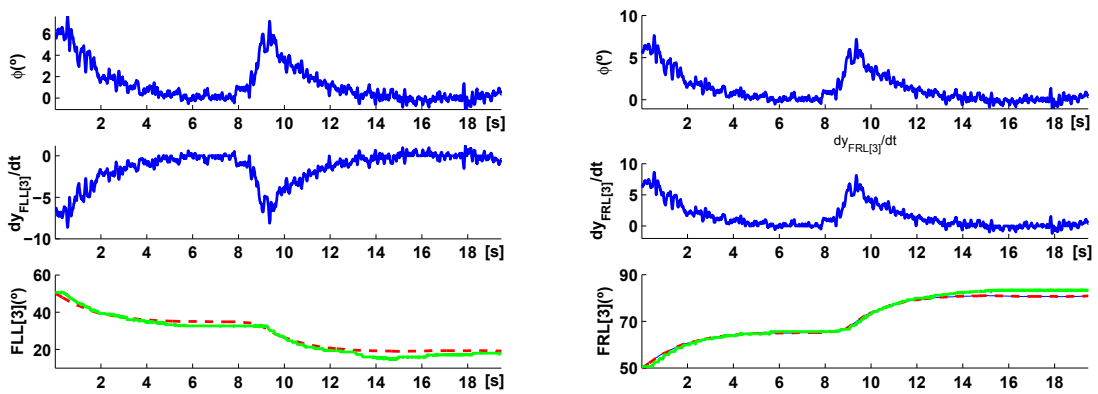

(a)

(b)

Figure 4: Real experiment. (a) Fore Left Knee (b) Fore Right Knee. Up panel: lateral tilt $(\phi)$; Middle panel: $\dot{y}_{\mathrm{i}[3]}$; Bottom panel: $\vec{x}_{\mathrm{i}[3]}$ (dashed line) and $\overrightarrow{\tilde{x}}_{\mathrm{i}[3]}$ (solid line) $(i=\mathrm{FLL}, \mathrm{FRL})$.

Fig. 5, depicts the obtained results. The robot successfully reacts to lateral tilt changes by reducing this to a minimum. The trajectories are symmetric for right and left legs. When the robot is inclined to the left (positive lateral tilt), $\dot{y}_{\mathrm{FLL}[3]}$ is negative, thus $y_{\mathrm{FLL}[3]}$ is reduced and the robot extends this leg. At $t \sim 8 \mathrm{~s}$, the robot is inclined to its right and lateral tilt becomes negative. Thus, $\dot{y}_{\mathrm{FLL}[3]}$ is positive and $y_{\mathrm{FLL}[3]}$ is increased meaning the fore left leg folds. Balanced position is achieved at $t \sim 12 \mathrm{~s}$
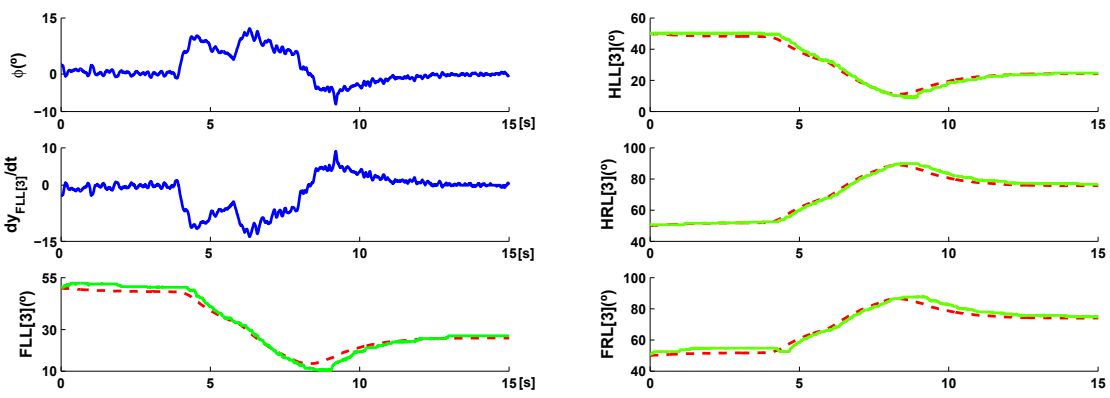

(a)

(b)

Figure 5: a) Fore Left knee. Up panel: lateral tilt $(\phi)$; Middle panel: $\dot{y}_{\mathrm{FLL}[3]}$; Bottom panel: planned $\vec{x}_{\mathrm{FLL}[3]}$ (dashed line) and $\overrightarrow{\tilde{x}}_{\mathrm{FLL}[3]}$ (solid line) trajectories. (b) Planned $\vec{x}_{\mathrm{i}[3]}$ (dashed line) and $\overrightarrow{\tilde{x}}_{\mathrm{i}[3]}$ (solid line) trajectories $(i=\mathrm{HLL}, \mathrm{HRL}, \mathrm{FRL})$.

\section{Conclusions}

In this article, we have presented a controller for correcting posture in an irregular terrain, where a MPG implemented as two embedded dynamical systems 
was able to generate discrete mode of movement. Online trajectory modulation is achieved through the inclusion of feedback loops that enable to take external perturbations into account (lateral tilt), such that when the environment changes, the system online adjusts the dynamics of trajectory generation. Moreover, due to the properties of dynamical systems the trajectory will always remain smooth.

As an application we apply a network of MPGs coupled together to the control of a quadruped robot (an ers7 AIBO). The robot was subjected to external perturbations that change its lateral tilts, measured by the built-in accelerometers. The MPGs network uses this information to compensate for the tilt changes and reduce them to near zero. The obtained results have been quite satisfactory. This controller showed to be fast enough since the robot can quickly recover from the induced physical inclinations. The controller also proved to be efficient according to the obtained results.

Presently, we are extending this work to compensate not only the lateral tilt but also the pitch inclination and merging both information in a single controller. We are also working on the integration of the touch sensors' information in order to assure contact of the robots' end effectors with the platform before performing a posture correction. Further, we are extending this work to combine this approach in order to obtain locomotion.

\section{References}

[1] J Pratt, C Chew, A Torres, P Dilworth, G Pratt, Virtual Model Control: An intuitive approach for bipedal locomotion, The Int. J. of Robotics Research, 2001, 20 (2), 129-143

[2] Y. Fukuoka, H. Kimura, and A. Cohen. Adaptive dynamic walking of a quadruped robot on irregular terrain based on biological concepts. Int. J. of Robotics Research, 3-4:187-202, 2003

[3] A Ijspeert, J Nakanishi, S Schaal, Learning attractor landscapes for learning motor primitives, Advances in Neural Information Processing Systems 15, 2003, 1547-1554

[4] F Delcomyn. Neural basis for rhythmic behaviour in animals. Science, 210:492-498, 1980

[5] S Giszter, F Mussa-Ivaldi, E Bizzi. Convergent force fields organized in the frog's spinal cord. J. of Neuroscience, 13:467-491, 1993

[6] G Taga, Emergence of bipedal locomotion through entrainment among the neuro-musculoskeletal system and the environment, Physica D, 1994, 75 (1-3), 190-208

[7] R Blickhan. The spring-mass model for running and hopping. J. Biomechanics, 22(11-12):1217-1227, 1989

[8] M Bühler, S Koditscheck. Planning and control of a juggling robot. Int J of Robotics Research, 13(2), 101-118, 1994

[9] S Degallier, C P. Santos, L Righetti and A Ijspeert - Movement Generation using Dynamical Systems: a Drumming Humanoid Robot. In Humanoids'06 IEEE-RAS International Conference on Humanoids Robots, Genova, Italy, December 4-6 2006

[10] Santos, C P; Ferreira, M; Oliveira, M; Pires, A; Dégallier, S and Ijspeert, A -Choreography generation for a quadruped robot using dynamical systems, Autonomous Robots (submitted)

[11] Vukobratovic, M., Borovac, B. Zero-moment point - thirty five years of life. In International Journal of Humanoid Robotics, 1(1), 157-173, 2004. 